
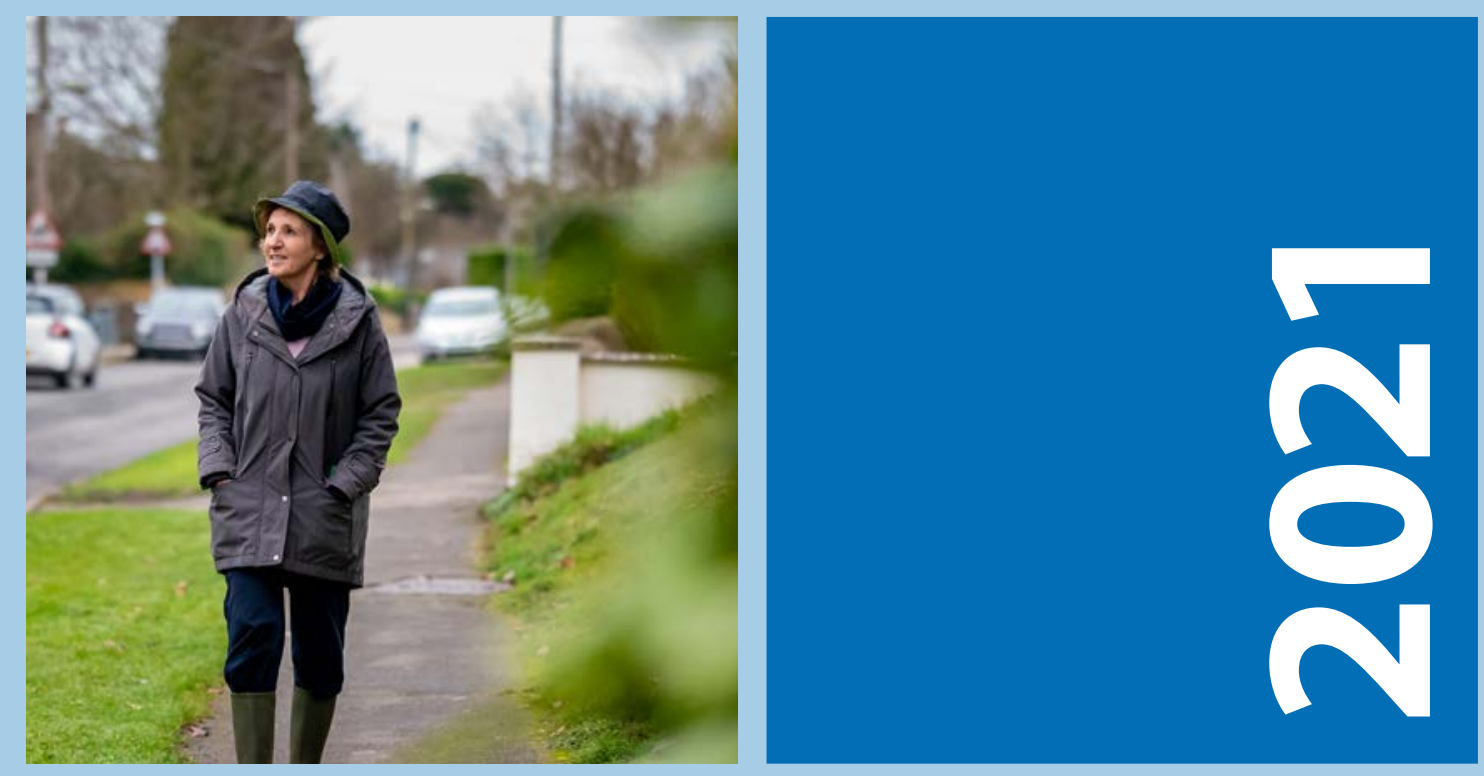

\title{
New self-reported exhaustion during the COVID-19 Pandemic
}

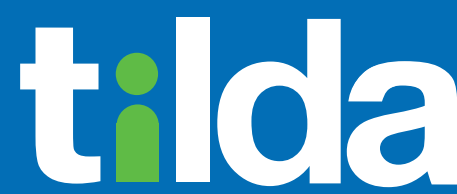

Staidéar Fadaimseartha na hÉireann um Dhul in Aois

The Irish Longitudinal Study on Ageing 


\section{New self-reported exhaustion during the COVID-19 Pandemic}

Ann Monaghan, Nollaig Bourke, Aisling O'Halloran, Mark Ward, Feng Xue, Glenn Jennings, Rose Anne Kenny and Roman Romero-Ortuno

On behalf of the TILDA team

February 2021 
Copyright $@$ The Irish Longitudinal Study on Ageing 2021

The Irish Longitudinal Study on Ageing

Trinity College Dublin

Dublin 2

Tel: +35318962509

Email: tilda@tcd.ie

Website: www.tilda.ie

https://www.doi.org/10.38018/TildaRe.2021-02 


\section{Acknowledgements}

We would like to acknowledge the vision and commitment of our study funders, the Department of Health, the Health Research Board, The Atlantic Philanthropies, Science Foundation Ireland and Irish Life plc. We would like to state that any views expressed in this report are not necessarily those of the Department of Health or of the Minister for Health. We would also like to thank the TILDA participants without whom this research would not be possible. 


\section{Table of Contents}

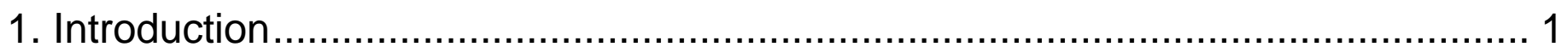

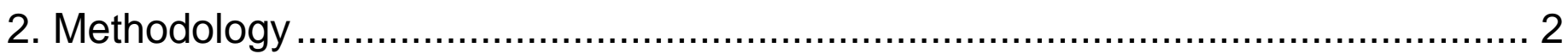

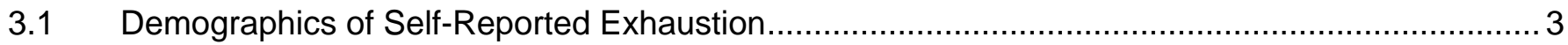

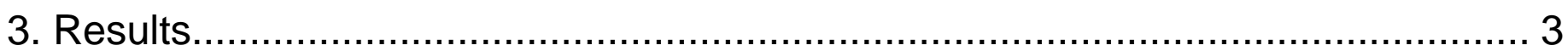

3.2 Self-Reported Exhaustion and COVID-19 Status ………......................................................

3.3 Variables Predicting New-Onset Self-Reported Exhaustion ……...........................................

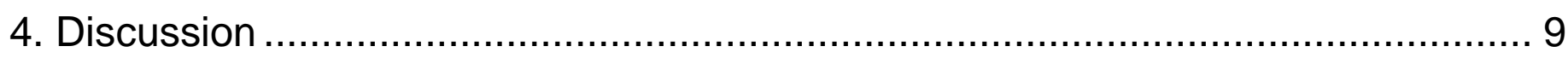

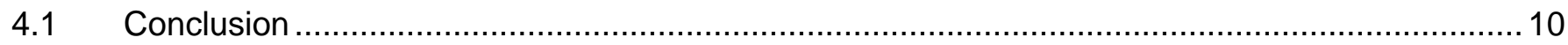

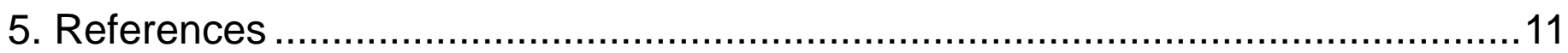




\section{Introduction}

In 2020, participants from The Irish Longitudinal Study on Ageing were surveyed to provide vital and timely information on lived experiences of older adults during the SARS-CoV-2 pandemic. During 2020, older adults experienced many challenges, such as living with restrictions on movement, having less opportunities for socialisation and, in some cases, limited access to healthcare [1]. Consequently, lifestyles are impacted, with both negative [2] and positive [3] effects being reported in the literature. This report concentrates on the prevalence of new-onset self-reported exhaustion (SRE) in 2020 amongst TILDA participants, and explores possible predictors and associated factors. The relationship with SARS-CoV-2 status and commonly reported COVID-19 symptomatology are also investigated. 


\section{Methodology}

Baseline data was extracted from TILDA Wave 5 (January-December 2018) and incident data from the dedicated TILDA COVID-19 questionnaire (July-November 2020). Self-reported exhaustion (SRE: everything I do is an effort and/or I can't get going) is a component of the frailty phenotype assessment undertaken by TILDA participants, as described by Fried et al [4]. New-onset self-reported exhaustion is determined by comparing responses to this assessment component in Wave 5 and in the COVID-19 questionnaire i.e. new SRE was indicated for those participants who self-report exhaustion in the COVID-19 questionnaire, but not in the previous Wave 5 assessment. Groups in this report are disaggregated by new SRE in 2020, versus no new SRE in 2020. Comparisons between groups are carried out with Chi-square tests or the 2-sided Mann-Whitney U-test. Independent predictors of new SRE are investigated with a multivariable binary logistic regression model. For each predictor, we report the Odds Ratio (OR) with $95 \%$ confidence interval $(\mathrm{Cl})$. The level of statistical significance is set at $\mathrm{P}<0.05$. 


\section{Results}

\subsection{Demographics of Self-Reported Exhaustion}

There are 5,225 Wave 5 and 3,876 COVID-19 Wave participants. The merged sample includes 3,554 participants (baseline mean age 69.0, 57\% women). Of those, 528 (15.6\%) have new SRE from the COVID-19 questionnaire, and 2,867 do not (Figure 1).

Figure 1. Percentage of merged cohort reporting new versus no new self-reported exhaustion in 2020

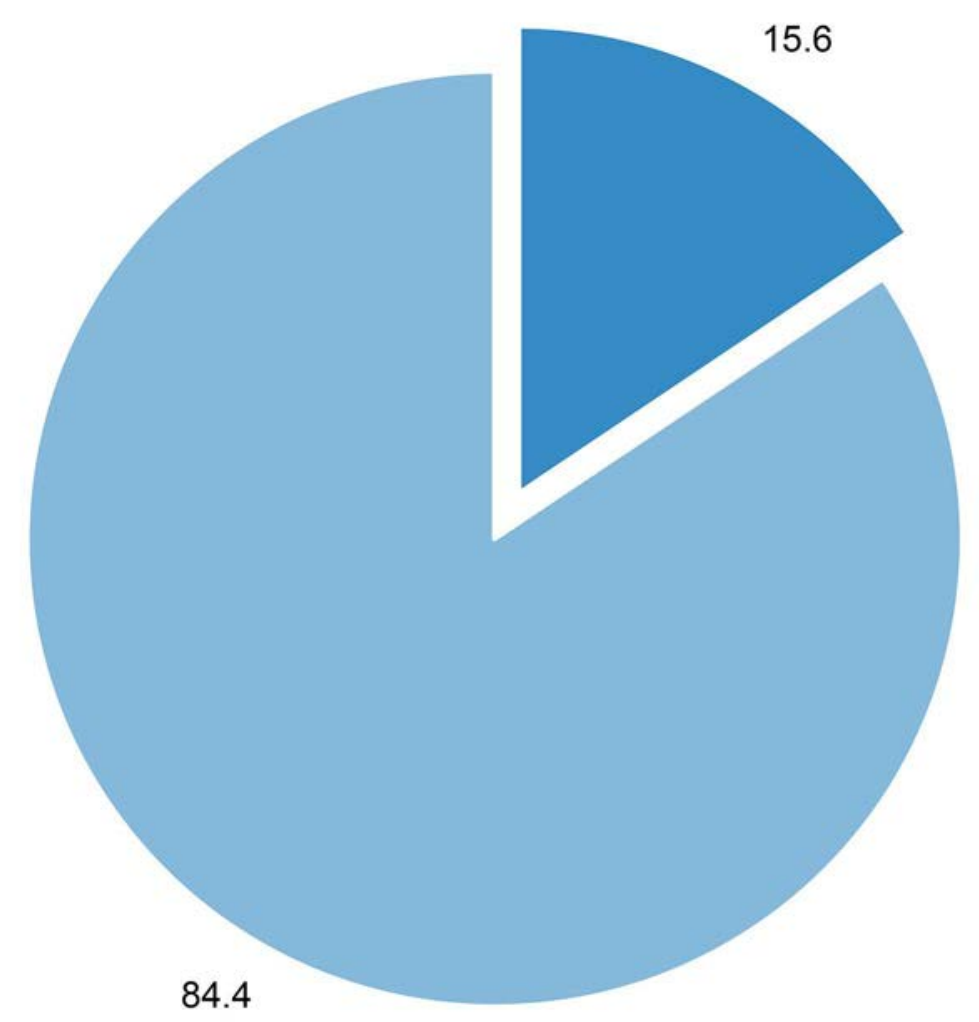

- New Exhaustion in $2020 \quad$ = No New Exhaustion in 2020 
Those reporting new SRE have a significantly higher mean age, and are proportionately more female, as described in Table 1. Such participants are also living with significantly more chronic illness and physical limitations.

Table 1. Demographic breakdown of groups, disaggregated by new self-reported exhaustion in 2020.

\begin{tabular}{l|c|c} 
& No New Exhaustion in 2020 & New Exhaustion in 2020 \\
& $(\mathrm{N}=\mathbf{2 8 6 7 )}$ & $69.9(8.8)$ \\
\hline $\begin{array}{l}\text { Age at Wave 5*, mean (SD) } \\
\text { Female sex, \% }\end{array}$ & $68.6(7.9)$ & 58.1 \\
\hline $\begin{array}{l}\text { Number of chronic conditions at Wave 5*, } \\
\text { median (IQR) }\end{array}$ & 56.3 & $2(2)$ \\
\hline $\begin{array}{l}\text { Number of regular medications at Wave 5*, } \\
\text { median (IQR) }\end{array}$ & $2(3)$ & $3(4)$ \\
\hline $\begin{array}{l}\text { Number of physical limitations at Wave 5*, } \\
\text { median (IQR) }\end{array}$ & $1(2)$ & $2(3)$ \\
\hline
\end{tabular}

*significant difference between groups

Participants with new SRE in 2020 have attained lower levels of education than participants reporting no new SRE in 2020, with proportionately more people experiencing new SRE reporting leaving school prior to or during second level education, as in Figure 2 below.

Figure 2. Education level of cohort reporting new versus no new self-reported exhaustion in 2020

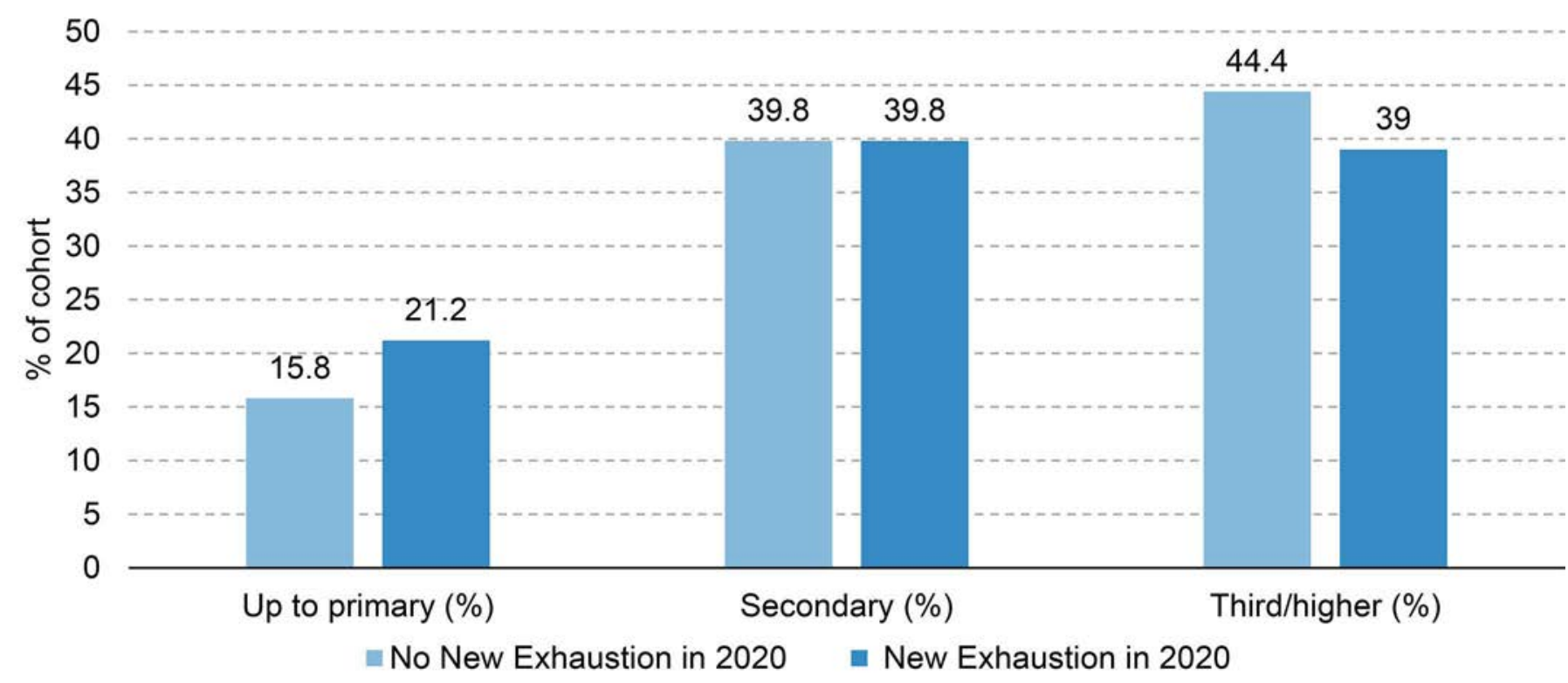


In addition, participants reporting new SRE in 2020 also have significantly worse self-reported emotional and/or mental health than those not reporting new SRE, as in Figure 3. There are significantly more participants with new SRE reporting their emotional/ mental health as 'fair' or 'poor', compared to those with no new SRE in 2020.

Figure 3. Self-reported mental/ emotional health of cohort reporting new versus no new self-reported exhaustion in 2020

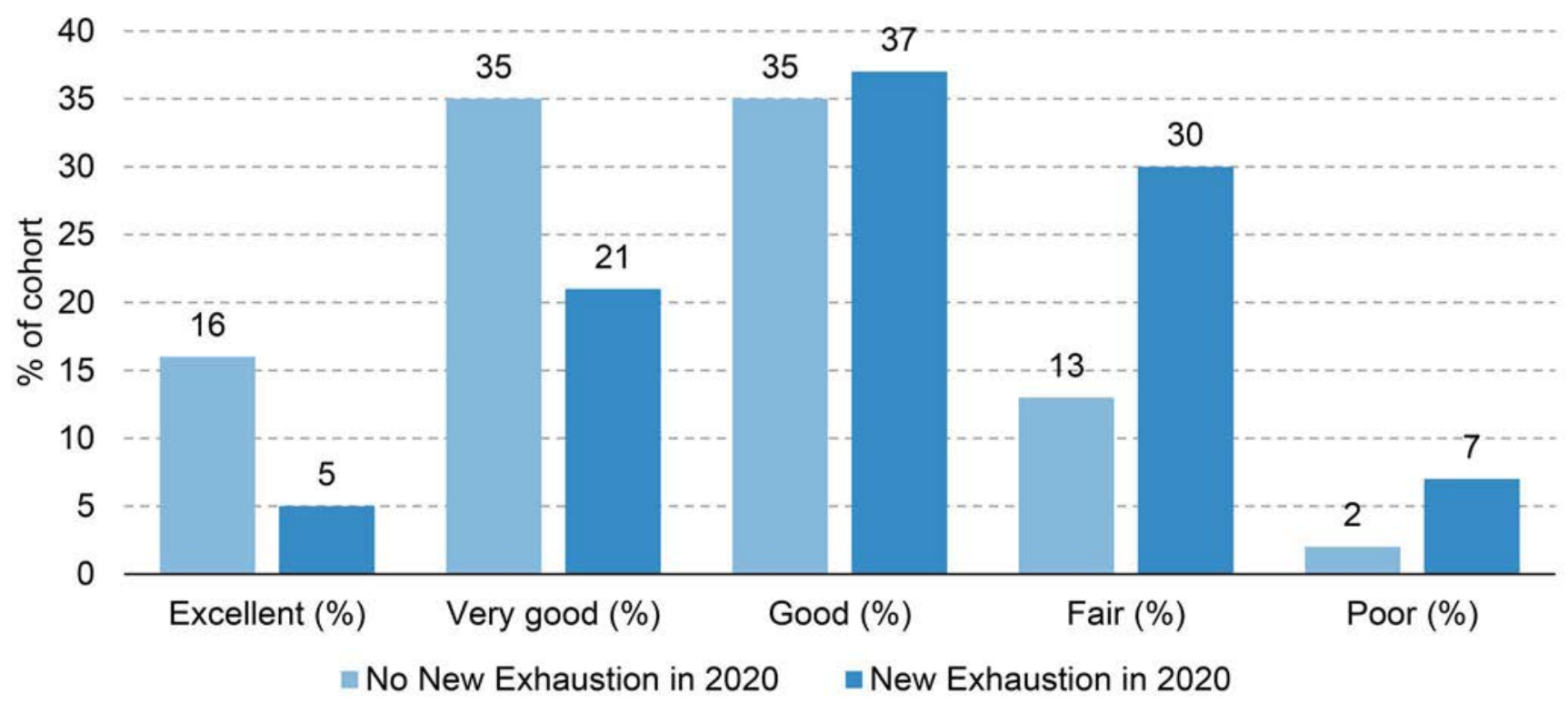

\subsection{Self-Reported Exhaustion and COVID-19 Status}

Participants with new-onset SRE in 2020 are comparatively more likely to report suspected or diagnosed COVID-19 (Figure 4) than those with no new SRE.

Figure 4. Percentage of cohort with suspected or diagnosed COVID-19 reporting new versus no new self-reported exhaustion in 2020

New Exhaustion in 2020

No New Exhaustion in 2020

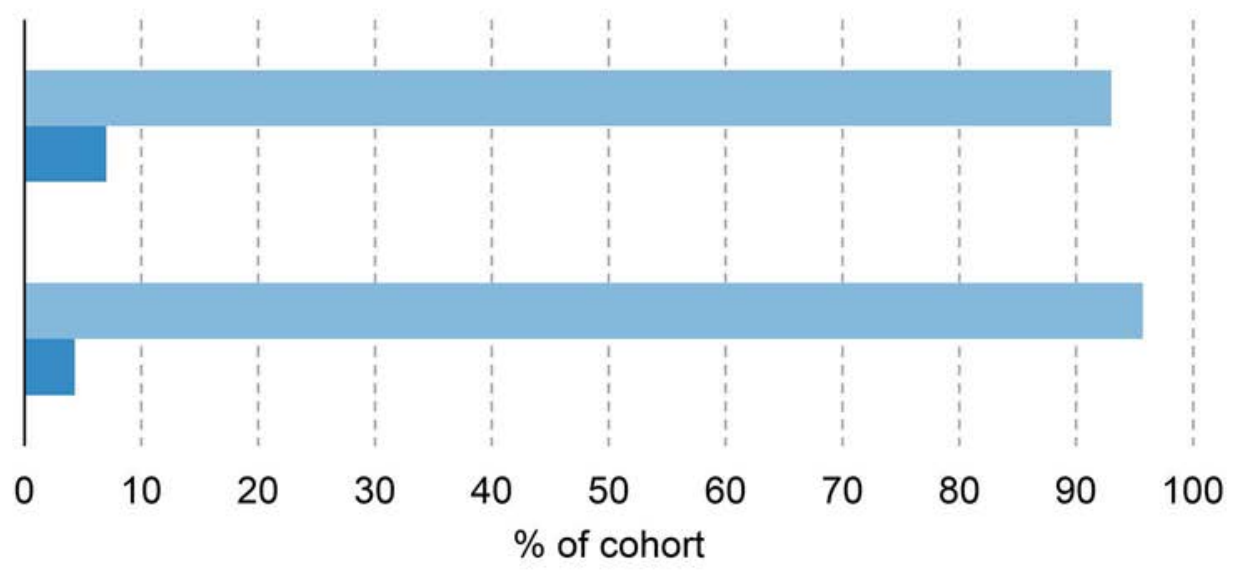

- COVID-19 not diagnosed or suspected 
When the nature of diagnosis is explored, this trend persists, where participants reporting new SRE in 2020 have proportionately more confirmed diagnoses of COVID-19, or tests confirming presence of SARS-CoV-2 (Figure 5). These findings are statistically significant.

Figure 5. SARS-CoV-2 Status of cohort reporting new versus no new self-reported exhaustion

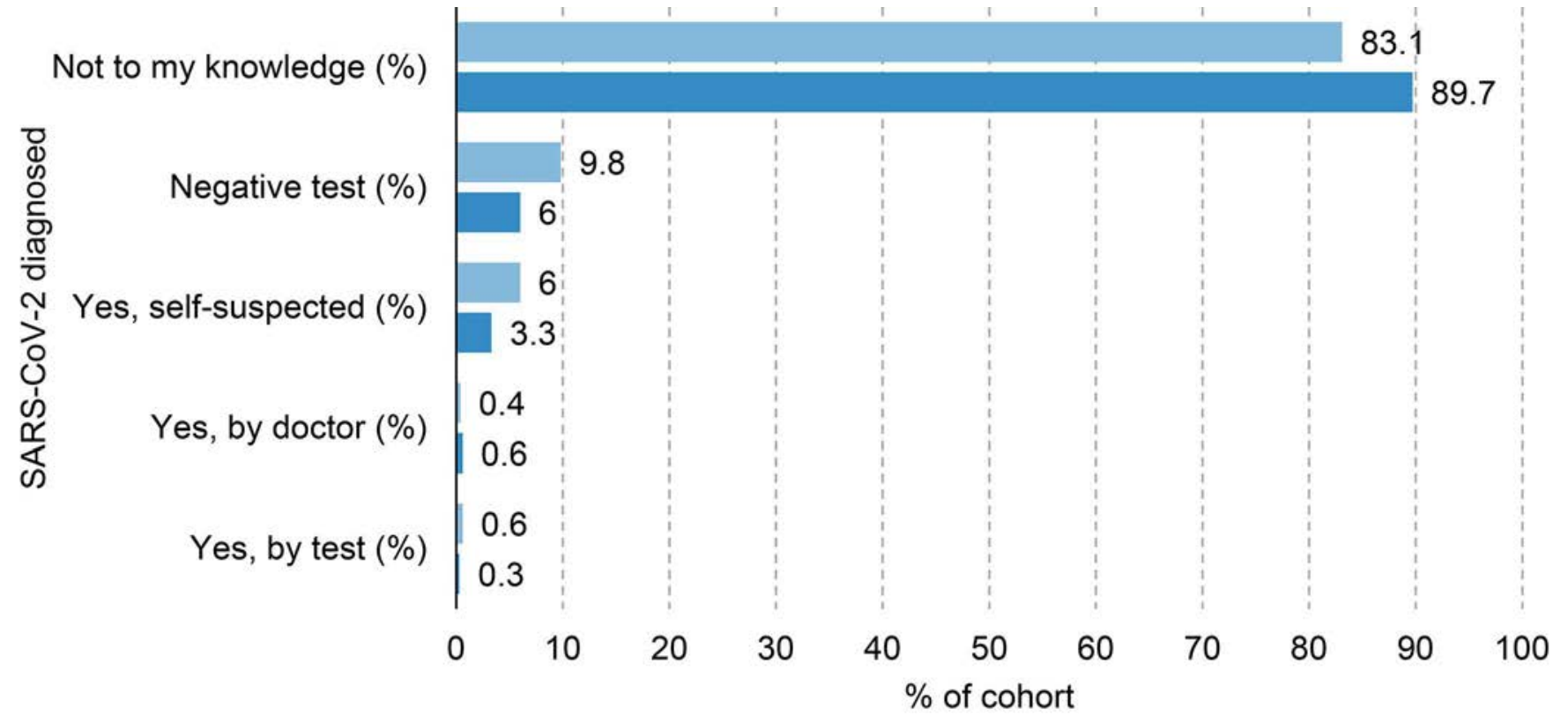

New Exhaustion in $2020 \quad$ No New Exhaustion in 2020

The proportion of participants experiencing commonly reported symptoms associated with COVID-19 are also explored. These data indicate that participants reporting new SRE in 2020 are experiencing proportionately more symptoms such as muscle and joint pain, breathlessness, cough, sore throat, diarrhoea and loss of taste or smell, as described in Figure 6. 
Figure 6. Commonly reported symptoms of COVID-19, as experienced by participants reporting new versus no new self-reported exhaustion

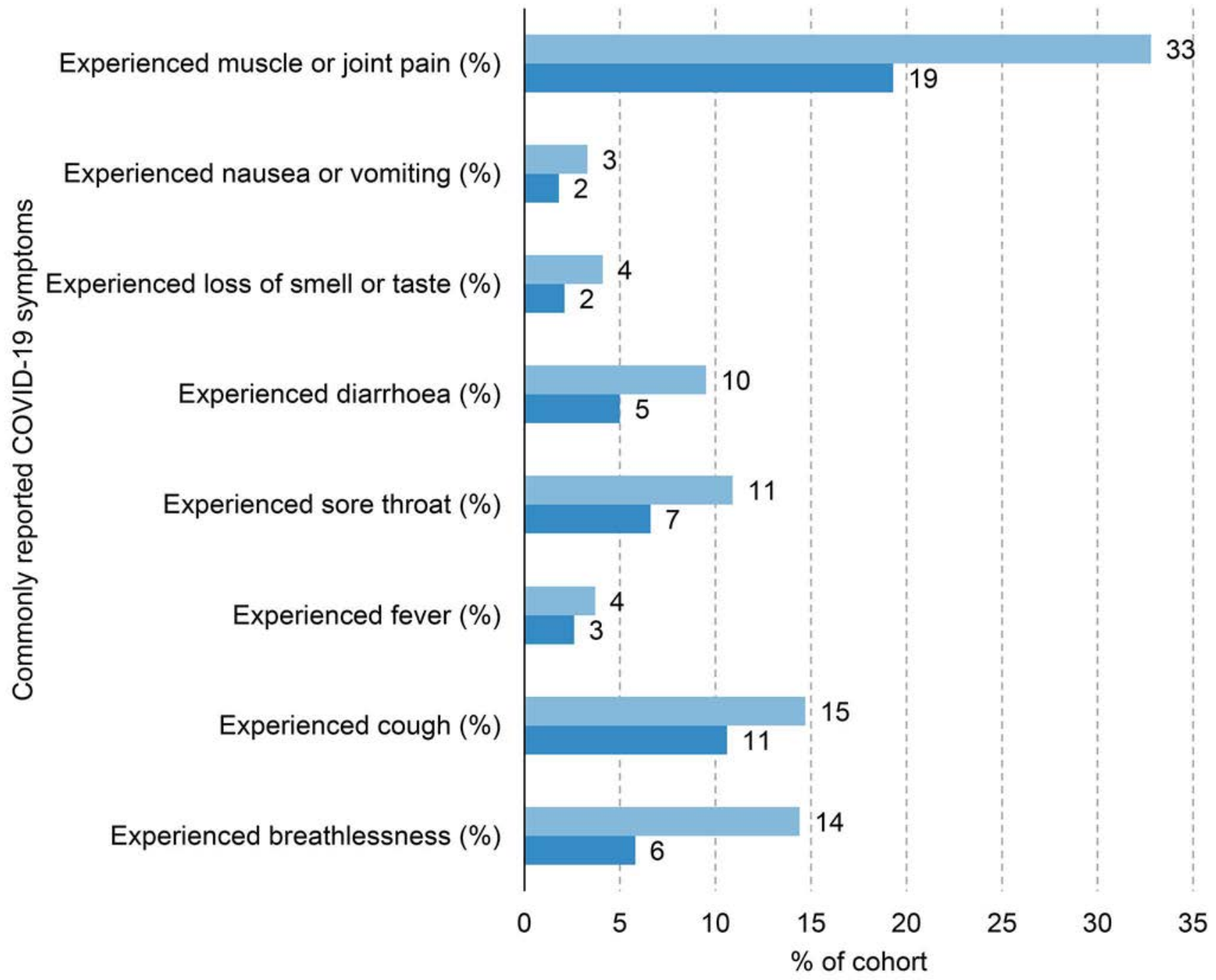

$\square$ New Exhaustion in $2020 \quad$ No New Exhaustion in 2020

\subsection{Variables Predicting New-Onset Self-Reported Exhaustion}

Independent predictors of new SRE were explored. The significant predictors were: (1) pre-pandemic number of physical limitations, (2) within-pandemic self-reported emotional/mental health (OR 1.83, 95\% Cl: 1.61-2.08, $\mathrm{P}<0.001$ ) and (3) experience of muscle or joint pain (OR 1.57, 95\% Cl 1.17-2.10, $\mathrm{P}=0.003$ ). SARS-CoV-2 infection, either diagnosed (by test or doctor) or self-suspected COVID-19 were not independent predictors of new SRE. 


\begin{tabular}{|c|c|c|c|c|}
\hline & \multirow{2}{*}{$\begin{array}{l}\text { Odds } \\
\text { Ratio }\end{array}$} & \multicolumn{2}{|c|}{ 95\% C.I. for Odds Ratio } & \multirow{2}{*}{$\mathbf{P}$} \\
\hline & & Lower & Upper & \\
\hline Baseline age & 1.01 & 1.00 & 1.03 & 0.140 \\
\hline Female sex & 0.99 & 0.77 & 1.27 & 0.929 \\
\hline Third/higher education & 0.90 & 0.70 & 1.14 & 0.364 \\
\hline Number of chronic diseases at TILDA Wave 5 & 0.92 & 0.83 & 1.03 & 0.141 \\
\hline Number of regular medications at TILDA Wave 5 & 1.01 & 0.96 & 1.07 & 0.591 \\
\hline Number of physical limitations at TILDA Wave 5 & 1.10 & 1.03 & 1.17 & 0.005 \\
\hline $\begin{array}{l}\text { Self-reported emotional/mental health during the } \\
\text { COVID-19 pandemic }\end{array}$ & 1.83 & 1.61 & 2.08 & $<0.001$ \\
\hline Experienced breathlessness & 1.53 & 0.91 & 2.57 & 0.106 \\
\hline Experienced cough & 0.76 & 0.48 & 1.20 & 0.236 \\
\hline Experience fever & 0.36 & 0.10 & 1.25 & 0.109 \\
\hline Experienced sore throat & 0.84 & 0.48 & 1.46 & 0.525 \\
\hline Experienced diarrhoea & 1.53 & 0.91 & 2.59 & 0.113 \\
\hline Experienced loss or taste/smell & 1.52 & 0.63 & 3.69 & 0.352 \\
\hline Experienced nausea/vomiting & 0.41 & 0.12 & 1.38 & 0.151 \\
\hline Experienced muscle or joint pain & 1.57 & 1.17 & 2.10 & 0.003 \\
\hline COVID-19 diagnosed or suspected & 1.56 & 0.88 & 2.76 & 0.131 \\
\hline
\end{tabular}

$\mathrm{Cl}$ : confidence interval. 


\section{Discussion}

Fatigue and exhaustion are debilitating symptoms which can negatively impact quality of life and lead to impaired function, especially when experienced in chronicity. The causes of fatigue are poorly understood, although it can be accompanied by various neurological, cardiovascular, respiratory, and gastrointestinal manifestations. Moreover, fatigue is a common symptom of viral infection, which can persist long after the initial infection is resolved [5]. Fatigue is therefore thought to be related to multi-system dysregulation [6, 7]. This exploration of new-onset self-reported exhaustion in the TILDA cohort concomitant to the 2020 SARSCoV-2 pandemic reveals a significant proportion of participants experienced a new-onset of exhaustion in 2020. Associations of new-onset exhaustion with severity of multimorbidity and physical limitations, and selfreported mental and emotional health are established.

Exhaustion is a commonly reported symptom of chronic disease [8, 9]. Thus, new-onset exhaustion could be attributable to increased burden of multimorbidity, associated with avoidance of healthcare facilities [10] and/or limited access to lifestyle management strategies for fatigue during periods of lockdown. Periods of stay-at-home orders are associated with negative health-related outcomes in chronic disease cohorts, as a consequence of reduced physical activity and increased psychosocial stress [2]. Indeed, a substantially increased prevalence of perceived stress and depressive symptoms is reported in 2020 in the TILDA cohort, which are thought to be linked to a fear of contracting SARS-CoV-2 virus and a perceived lack of control of one's own environment and wellbeing [11, 12].

This increased prevalence of perceived stress and depressive symptoms may explain the association of new-onset self-reported exhaustion with worse self-reported emotional/mental health, as demonstrated in this report. It is well established that anxiety, depression and fatigue frequently manifest concurrently [13]. Individuals with either fatigue or depression have an approximately two-fold increased risk for comorbid presentation of both traits, compared to the general population [14].

An additional predictor for new-onset self-reported exhaustion is muscle and joint pain. Inflammation is proposed as a possible aetiology of both depression and fatigue [15], which may also explain the evident relationship between pain and fatigue in this cohort. Associations between fatigue and inflammation are established in chronic disease cohorts such as rheumatoid arthritis [16]. Neuro-inflammation, associated with viral infection [5] may also drive pain and fatigue symptoms. Moreover, pain itself is thought to instigate depressive symptoms, with chronic pain and chronic exhaustion displaying significant overlap in manifestations and pathogenesis [17].

As these data are captured against a backdrop of the SARS-CoV-2 pandemic, an investigation of SARS- 
CoV-2 status and common COVID-19 symptomatology is also explored. Participants experiencing newonset exhaustion have a higher prevalence of suspected or diagnosed SARS-CoV-2 and self-reported COVID-19 symptomatology in comparison to participants with no new exhaustion. Post-viral infection fatigue is a well-established phenomenon [5], and is highly prevalent in COVID-19. Up to 53\% of COVID-19 patients experience symptoms consistent with severe fatigue a median of 10 weeks after their initial illness [18]. Survivors of previous coronavirus-related illnesses such as the Severe Acute Respiratory Syndrome (SARS) in 2003 and the Middle Eastern Respiratory Syndrome (MERS) in 2012 have been documented to suffer from myalgia (muscle pains), depression, chronic fatigue and disordered sleep up to three years after initial infection [19]. Thus, further exploration of the relationship between new-onset self-reported exhaustion and SARS-CoV-2 status is warranted. Future TILDA reports will explore the relationship between exhaustion and the results of a new study, entitled 'SABS-TILDA: SARS-CoV-2 specific AntiBodieS in The Irish Longitudinal Study on Ageing (TILDA)'. This study, led by Dr Nollaig Bourke and Professor Rose Anne Kenny, will provide an important opportunity to assess the prevalence of SARS-CoV-2 infection in the TILDA cohort, and will thus retrospectively inform earlier collected data on COVID-19 manifestations, including self-reported exhaustion.

\subsection{Conclusion}

This report examines the prevalence of new-onset self-reported exhaustion in TILDA participants using the dedicated COVID-19 questionnaire. $15.6 \%$ of participants reported new- onset SRE, associated with greater degree of multimorbidity, physical limitations and worse self-reported mental/emotional health. In particular, older people with pre-existing physical limitations have been significantly burdened by the pandemic. Our results suggest that a multidisciplinary rehabilitative approach to psychological support, pain management, and multimorbidity intervention may help reduce self-reported exhaustion in older people during the pandemic. 


\section{References}

1. Blumenthal, D., et al., Covid-19 - Implications for the Health Care System. New England Journal of Medicine, 2020. 383(15): p. 1483-1488.

2. Cransac-Miet, A., et al., Impact of COVID-19 lockdown on lifestyle adherence in stay-at-home patients with chronic coronary syndromes: Towards a time bomb. International Journal of Cardiology, 2021. 323: p. 285-287.

3. Naqvi, H.R., et al., Improved air quality and associated mortalities in India under COVID-19 lockdown. Environmental Pollution, 2020.

4. Fried, L.P., et al., Frailty in older adults: evidence for a phenotype. J Gerontol A Biol Sci Med Sci, 2001. 56(3): p. M146-56.

5. Yamato, M. and Y. Kataoka, Fatigue sensation following peripheral viral infection is triggered by neuroinflammation: who will answer these questions? Neural regeneration research, 2015. 10(2): p. 203204.

6. Katz, B.Z., et al., The International Collaborative on Fatigue Following Infection (COFFI). Fatigue : biomedicine, health \& behavior, 2018. 6(2): p. 106-121.

7. Cortes Rivera, M., et al., Myalgic Encephalomyelitis/Chronic Fatigue Syndrome: A Comprehensive Review. Diagnostics (Basel, Switzerland), 2019. 9(3): p. 91.

8. Flensner, G., A.C. Ek, and O. Söderhamn, Lived experience of MS-related fatigue--a phenomenological interview study. Int J Nurs Stud, 2003. 40(7): p. 707-17.

9. Stone, P., M. Richards, and J. Hardy, Fatigue in patients with cancer. European Journal of Cancer, 1998. 34(11): p. 1670-1676.

10. Czeisler, M.É., et al., Delay or avoidance of medical care because of COVID-19-related concernsUnited States, June 2020. Morbidity and mortality weekly report, 2020. 69(36): p. 1250.

11. Ward, M. and R.A. Kenny, Perceived stress before and during the COVID-19 pandemic. The Irish Longitudinal Study on Ageing. https://doi.org/10.38018/tildarb.2020-03, in The Irish Longitudinal Study on Ageing. 2020, The Irish Longitudinal Study on Ageing.: Dublin 
12. Ward, M., P. O'Mahoney, and K. R.A., Altered lives in a time of crisis: The impact of the COVID-19 pandemic on the lives of older adults in Ireland. Findings from The Irish Longitudinal Study on Ageing. , in The Irish Longitudinal Study on Ageing. 2021.

13. Lallukka, T., et al., Co-occurrence of depressive, anxiety, and somatic symptoms: trajectories from adolescence to midlife using group-based joint trajectory analysis. BMC Psychiatry, 2019. 19(1): p. 236.

14. Corfield, E.C., N.G. Martin, and D.R. Nyholt, Co-occurrence and symptomatology of fatigue and depression. Comprehensive psychiatry, 2016. 71: p. 1-10.

15. Lee, C.-H. and F. Giuliani, The Role of Inflammation in Depression and Fatigue. Frontiers in Immunology, 2019. 10(1696).

16. Madsen, S.G., et al., Correlations between fatigue and disease duration, disease activity, and pain in patients with rheumatoid arthritis: a systematic review. Scand J Rheumatol, 2016. 45(4): p. 255-61.

17. Clauw, D.J. and G.P. Chrousos, Chronic pain and fatigue syndromes: overlapping clinical and neuroendocrine features and potential pathogenic mechanisms. Neuroimmunomodulation, 1997. 4(3): $p$. 134-153.

18. Townsend, L., et al., Persistent fatigue following SARS-CoV-2 infection is common and independent of severity of initial infection. medRxiv, 2020: p. 2020.07.29.20164293.

19. Moldofsky, H. and J. Patcai, Chronic widespread musculoskeletal pain, fatigue, depression and disordered sleep in chronic post-SARS syndrome; a case-controlled study. BMC Neurology, 2011. 11(1): p. 37. 